\title{
Newton per Meter
}

National Cancer Institute

\section{Source}

National Cancer Institute. Newton per Meter. NCI Thesaurus. Code C70474.

A unit of surface tension defined as the stretching force required to form a liquid film,

tending to minimize the area of a surface, equal to one newton per unit length of the film equal to one meter. 